\title{
Smoke impacts from prescribed burning in Victoria; developing a risk climatology
}

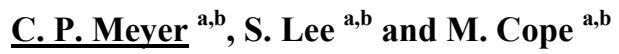 \\ ${ }^{a}$ CSIRO Division of Marine and Atmospheric Research, Private Bag No. 1 Aspendale 3195 Victoria \\ ${ }^{b}$ Bushfire Cooperative Research Centre, Albert St, East Melbourne, Victoria. \\ Email: mick.meyer@csiro.au
}

\begin{abstract}
Following an increase in the frequency of extreme fire weather in recent years and the subsequent loss of life and damage to property, fire agencies in Australia are under pressure to increase fuel reduction burning. However, at the same time, there is an increasing pressure to reduce the risk of population exposure to smoke. Reconciling these competing demands is a major challenge.
\end{abstract}

Currently, wind speed and direction are the main guides used by agencies for estimating regions at risk from smoke plume strikes. However, plume dispersion models and computing resources are now sufficiently developed so that the risk to towns and cities from planned fuel reduction and agricultural fires can also be assessed using these tools. This is of particular relevance in north central Victoria, Gippsland and the Riverina where there is a need to identify those areas of forest and farmland most likely to pose a smoke hazard to nearby towns during the autumn and spring burning seasons.

In this paper, we introduce a numerical modelling system which can be used to undertake an assessment of the risk to communities resulting from smoke generated by fuel reduction burning. The paper demonstrates how the system can be used in an inverse modelling mode to investigate the relationship between sensitive receptor- and upwind source regions. Using the Ovens Valley in north-eastern Victoria as an example, we apply the CSIRO dispersion model, The Air Pollution Model ( TAPM) coupled to the CSIRO Chemical Transport Model (CTM) to simulate the dispersion of PM2.5 emitted daily between 11:00 and 16:00 during April 2009 from each $3 \mathrm{~km} \times 3 \mathrm{~km}$ grid cell in a 50 x 50 cell domain centred on Harrietville. From these data we can assess the relative impact of each source cell on any receptor cell within the domain. Taking the towns of Myrtleford, Harrietville and Mt Beauty as test cases, we find that the greatest likelihood of smoke impact is from fires close to the receptor cell, however more distant sources are also significant, with the strongest located on the valley slopes. Vegetated source areas in the bottom of the valleys and on ridges have least impact. Harrietville and Mt Beauty, which lie in different valleys, nevertheless have similar source risk distributions, in contrast to Myrtleford, which lies downstream of Harrietville on the Ovens River, and has a totally different source risk profile. Significantly, there is no indication of a prevailing flow for any of the three receptor cells. We discuss how the system can be used to provide a self-consistent framework for testing smoke transport screening approaches for use by fire managers for planning prescribed burning schedules.

Keywords: Fuel reduction burning, transport modelling, screening modelling, smoke hazard, screening tools 
Meyer et al., Smoke impacts from prescribed burning in Victoria; developing a risk climatology.

\section{INTRODUCTION}

In Southern Australian forests, unplanned fires (commonly called wildfires) frequently have catastrophic effects on the land, property and people. Recent extreme events were the Black Saturday fires that devastated over 450,000 ha and killed 173 people. A Royal Commission into the disaster concluded that one of the contributing factors was the heavy fuel load present in the areas burnt, and consequently recommended that the annual rate of fuel reduction burning should increase to $5 \%$ of total State managed forest area (Teague et al., 2010). However, while low intensity, prescribed burns pose minimal risk to property, they produce large amounts of smoke. If this smoke fumigates local residents or sensitive agricultural crops, particularly grapes, then the risks to health or the risks of financial loss could be substantial. Therefore, increasingly, fire management authorities will be faced with the challenge of balancing the wildfire risk to communities against the risk to health and livelihood from the smoke generated by prescribed burns.

The principal pollutant of concern for health generated by wood combustion is fine (less than $2.5 \mathrm{um}$ ) particles; although still the subject of intensive investigation, the general risk for mortality is now well established (Pope and Dockery, 2006). The morbidity risks are also becoming clearer: exposure to smoke has been found is to increase the risks from asthma and cardio respiratory disease (Johnston et al., 2007). The risk to vineyards from smoke taint is a particularly vexed issue, occasionally leading to litigation (DEC, 2010). Although not yet confirmed, the pollutants of concern in this instance is thought to be a range of semi-volatile guaiacol compounds, which are phenolic glycosides (Kennison, 2009).

Fuel reduction burns are designed to be of low intensity (to avoid or minimize ecosystem damage) and to be contained within set boundaries. (Fire Ecology Working Group, 2004). Hence the conditions under which these prescribed fires can occur are specified as light winds and wind gusts, low temperature, and moderate humidity. However, these conditions also limit the ventilation rate and smoke dispersion and, therefore, promote the build-up of air pollution. The season for fire management burns is limited by the availability of suitable conditions;, particularly dry fuel and stable weather patterns. Such conditions have a maximum likelihood of occurring during the autumn months, and, with a lower probability, during spring to early summer. The window for autumn prescribed burning is also widening, and now sometimes extends into the early winter when the smoke emissions add to those of domestic wood heaters, further exacerbating the pollution impacts.

Because the requirement to undertake extensive fuel burns can only occur under conditions that maximize air pollution, there is an imperative to select conditions in which smoke plumes will not pass over populated areas and to provide advance warning to potentially effected populations, so that appropriate steps can be taken to minimize population exposure to smoke. This involves planning on several timescales. At the operational level, detailed smoke dispersion forecasting in the days preceding planned burns, can enable fire managers to choose time and patterns of burning that limit the smoke exposure risks. However, dispersion forecasting is technically challenging, time consuming and computationally intensive. Consequently planning currently relies heavily on fire managers' knowledge and experience of smoke dispersion in order to design burning patterns and schedules that allow for adequate smoke dispersion.

Smoke dispersion models can assist planners without the need for on-line modelling. By determining the general patterns and the climatology of smoke dispersion for defined regions, models can aid managers to identify coupes in the forests surrounding regional towns that pose the greatest risk of smoke impact. In this paper we provide an example of how a smoke dispersion modelling tool can be used to map smoke impact risk in the Ovens Valley of north east Victoria.

\section{METHODOLOGY}

The methodology leverages the capability of a coupled numerical weather and chemical transport modelling system. Figure 1 shows a schematic diagram of the modelling system, and indicates the major components: a) a meteorological modelling module; b) an emissions modelling module; c) a chemical transport modelling module and d) post processing module. All components of this system have been used extensively to model fires in Australia (e.g. Meyer et al., 2008, Meyer et al., 2011, Meyer et al., 2012). For the current project, the meteorological modelling was undertaken using TAPM (Hurley et al., 2009), a nestable, three-dimensional Eulerian numerical weather and air quality prediction system. This model was used to generate meteorological fields which including wind velocity, temperature, water vapour mixing ratio (including clouds), radiation and turbulence. The air quality component of TAPM, which includes a simple chemical transformation mechanism, was not used in the current study. 
Meyer et al., Smoke impacts from prescribed burning in Victoria; developing a risk climatology.

The smoke emissions modelling is a simplified version of that used by Meyer et al. (2008) to generate fuel loadings for the Top End of Australia. In brief, the burned areas are first identified using either remote sensing products such as area burned and fire spots or by on-ground monitoring by fire agencies, then combined with estimated fuel loads and emission factors. Information on diurnal variation is estimated from modelled rate of fire spread, using either a fire danger index, or a fire spread model. The spatial distribution of fuel loading is derived from a biogeochemical carbon cycle model, BIOS 2, which relates the drivers of production (intercepted radiation, temperature, soil moisture, rainfall, and vegetation class) to biomass and soil pools of carbon (Haverd et al., 2013).

The smoke dispersion modelling was undertaken using the CSIRO Chemistry Transport Model (CTM; Cope et al. 2004). The CTM is a three-dimensional Eulerian chemical transport model with the capability of modelling the emission, transport, chemical transformation, wet and dry deposition of an arbitrary number of gaseous and aerosol species. For the current study the CTM was configured to model multiple elemental carbon tracers which are used to represent an array of potential fire sources.

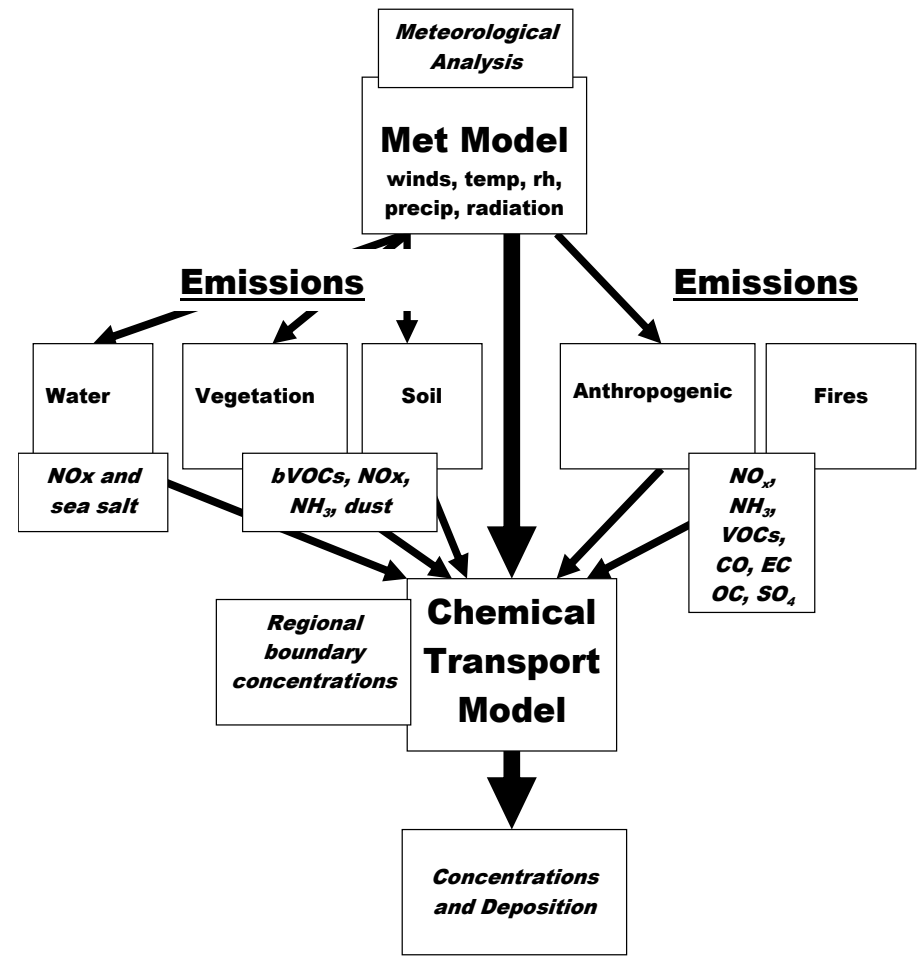

Figure 1. Schematic diagram summarising the smoke modelling system. VOC:volatile organic carbon; bVOC: biogenic component of the same; EC: elemental carbon; OC: organic carbon; $\mathrm{NO}_{\mathrm{x}}$ : oxides of nitrogen; $\mathrm{NH}_{3}$ : ammonia; $\mathrm{SO}_{4}$ : sulfate.

This system provides a self consistent framework for testing simplified methodologies that potentially can be used in the field (or from an operational centre) to assess the likelihood of smoke from a fuel reduction burn impacting on a sensitive receptor area. The methodology is self consistent because the predicted smoke transport is forced by the same prognostic meteorology that can then be used to test a hierarchy of approaches. In particular, the CTM system can be used to assess the following:

a. Whether the plume will be transported in the direction of a sensitive receptor by using near surface wind speed and direction at the fire location (or remote from the location of the fire);

b. The plume transport path using a forward trajectory calculation (either from wind observations or weather model predictions (Wain and Mills, 2005);

c. Forecasts plume transport over the next $24-48 \mathrm{~h}$ using a tracer transport model together with a numerical weather prediction;

d. Generation of $24-48 \mathrm{~h}$ forecasts for all significant air pollutant components of a fire using the 3dimensional CTM coupled with quantified fire emission estimates and numerical weather predictions; 
Meyer et al., Smoke impacts from prescribed burning in Victoria; developing a risk climatology.

e. Rank the likelihood that areas targeted for fuel reduction burns will cause plume strikes at the locations of sensitive receptors by using inverse modelling with tagged tracers) that can span from short term (hours) to climatology time scales (multiple years).

\section{EXAMPLE- DEVELOPMENT OF A RISK CLIMATOLOGY}

The inverse modelling methodology is demonstrated for April 2009. This has been selected because it is a typical month (and year) where fuel reduction burning took place in the Ovens Valley region. Application of the inverse modelling approach involves a four-step process.

\subsection{Step 1- Meteorological Modelling}

Meteorological fields were generated using TAPM configured with three nested grids (each 50 x 50 cells centred on the Ovens Valley) with cell spacings of 27,9 , and $3 \mathrm{~km}$. The purpose of using this system of nested domains is to downscale from synoptic scale weather patterns (which drive the $27 \mathrm{~km}$ spaced domain) to the weather at a grid spacing of $3 \mathrm{~km}$ over the Ovens Valley region. The large scale weather patterns are provided by the Bureau of Meteorology for April 2009. The model was configured with 25 vertical levels centred on the following heights (m): 10, 25, 50, 100, 150, 200, 250, 300, 400, 500, 600, 750, 1000, 1250, $1500,1750,2000,2500,3000,3500,4000,5000,6000,7000,8000$, thus providing the ability to simulate vertical shear in the wind, temperature and turbulence fields and the resultant effect on smoke plume transport. TAPM generates 3-dimensional meteorological fields which are stored at hourly intervals for input into the chemical transport model.

\subsection{Step 2- Emissions Modelling}

In order to investigate source/receptor relationships for smoke plumes in the Ovens Valley we generated fire emission fields for the study domain and simulation period. However, to develop a risk climatology we are primarily interested in the relative contribution of smoke from different regions on sensitive receptors. Thus we simplify the emission model described above and assume smoke emissions are proportional to the local fine-fuel loading. We assume ignition occurs between 11:00 and 16:00 and combustion is complete by 17:00.

Figure 2A shows the spatial distribution of fine fuel, estimated with Bios II, for the $3 \mathrm{~km}$ grid spacing. Fuel load varies from $0-4 \mathrm{t}$ carbon $\mathrm{ha}^{-1}$ in the north-west of the domain to $16 \mathrm{t}$ carbon $\mathrm{ha}^{-1}$ in the south-east. We also assume that the combustion conditions (which are a function of fuel dryness and meteorology), do not vary strongly across the modelling domain. However, this assumption can be relaxed in future scenarios to include the effect of local variations in fuel dryness and weather on fuel combustion and smoke emission characteristics as the system evolves further.

\subsection{Step 3- Dispersion Modelling}

The third step of the inverse modelling procedure involves tracking the individual emissions from each model cell in the $3 \times 3 \mathrm{~km}^{2}$ domain and calculating the plume dispersion. This is done for each hour of the day for the entire month. Cell-level emission tracking is achieved by tagging the emissions from each cell with a unique tracer. In order to track the individual emissions from a 50 x 50 cell $3 \mathrm{~km}$ domain, 2500 unique tracers are required. Although this can be done with a single simulation of the CTM, in practice we configure the CTM chemistry to use 100 individual tracers and then simulate the entire domain using 25 parallel runs on a computational cluster. Using this approach, the entire simulation process can be completed in just a few hours.

The characteristics of the tracer emissions are configured to be equivalent to PM2.5; to be unreactive, and thus an analogue of the elemental carbon component of the PM2.5 emissions. Thus we do not consider the generation of secondary aerosols; this will produce a slight underestimate of PM2.5 mass during lowventilation, precipitation-free multi-day events in which smoke is recycled through the airshed allowing photochemical conversion of gas-phase products into semi-volatile aerosol products. This can be considered further as the system evolves.

An additional consideration is the plume rise of the fire, resulting from the buoyancy forces in the hot smoke plumes. Here we assume that the fuel reductions burns have sufficient buoyancy to allow plume rise, but without sufficient energy to enable the plume to penetrate the stable layer at the top of the convective boundary layer (CBL). Thus the smoke plume becomes well mixed through the CBL after a few convective time-scales ( $\mathrm{h} / \mathrm{w}^{*}$; where $\mathrm{h}$ is the CBL height and $\mathrm{w}^{*}$ is the convective velocity scale). This typically leads to a well mixed state over the order of an hour for late summer/autumn conditions in mid-latitude regions. 
Meyer et al., Smoke impacts from prescribed burning in Victoria; developing a risk climatology.

Figure 2B demonstrates how this modelling configuration works. In this example we can see how, at hour 17 on $10^{\text {th }}$ April, the emissions from three separate source locations (Myrtleford, Harrietville, Mt. Beauty) are tracked by the model as they are advected downwind to the south-east within a north-westerly airflow. At any point within the airshed, the total primary particle mass loading can now be calculated by summing the individual contribution from each of the tagged tracer concentrations.
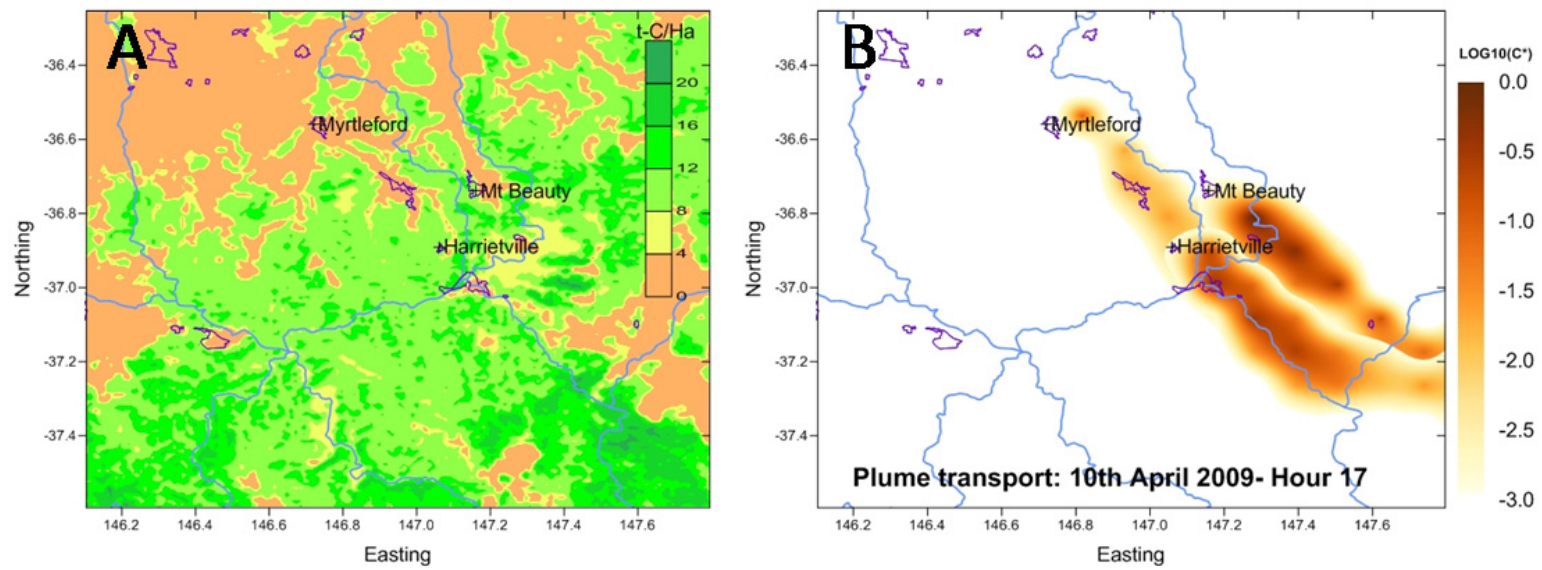

Figure 2. A: The spatial distribution of fuel load (tonnes of carbon per hectare) used to generate emissions for the smoke dispersion modelling. B: Plume transport from three tracers released at locations close to Myrtleford, Mt Beauty and Harrietville. Emission rates are proportional to the local fuel loading. Surface concentrations have been normalised relative to the maximum concentration in the grid and are expressed on a logarithmic scale.

\subsection{Step 4- Source Attribution using Inverse Modelling}

We are able to use tagged tracer species to track the individual plumes of each fuel reduction burn, and to sum these to calculate the total primary particle loading within the airshed. However this approach also allows the converse (inverse) question to be answered. That is, given the total primary particle concentrations at a receptor point, what is the relative contribution of each source region within the airshed to this total? This is demonstrated in Figure 3 where we show the smoke plumes from sources in three different source locations in the Ovens Valley region impacting the fine particle loading at Myrtleford during April 2009. Firstly it can be seen that a local fire source has the potential to impact with a high frequency within the local region: with the day-to-day variation in concentration resulting from variations in local meteorology. For sources further afield (Mt. Beauty and Harrietville), both the frequency and magnitude of plume impacts in Myrtleford are significantly reduced (Harrietville being a more significant source than Mt Beauty). This is a result of the reduced frequency of wind directions which lead to transport of the far-field plumes to Myrtleford, the increased levels of dispersion, and variations in the fuel loading at the source locations.

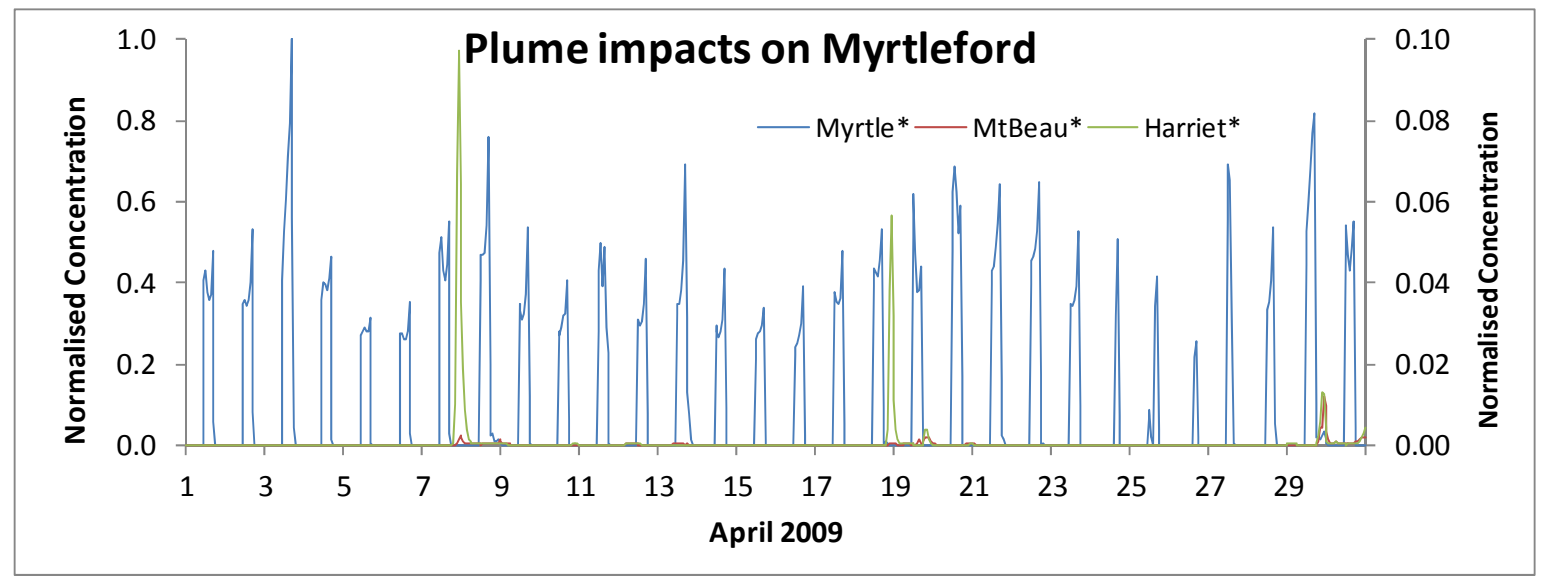

Figure 3. Time series of normalised smoke plume impacts at Myrtleford for fires burning in the vicinity of Myrtleford (Myrtle*, left axis); at Mt Beauty (MtBeau*, right axis); at Harrietville (Harriet*, right axis). 
The results shown in Figure 3 can be generalised to consider the impact on Myrtleford of emissions from any location within the study region. This is demonstrated in Figure 4A, which quantifies how the emissions from any location on the $3-\mathrm{km}$ modelling domain contributes to the total primary fire particle loading at Myrtleford. It can be seen that, as shown in

, local sources have the largest impact on Myrtleford, and that there is a favourable transport path from the south-east which encompasses Harrietville, but not Mt. Beauty; hence explaining the difference between the level of impact between these two source regions (Figure 3). A similar analysis can be applied to other receptor locations such as Mt Beauty (Figure 4B) and Harrietville (Figure 4C). Both sites have source contribution patterns that are broadly similar, which is promising from the perspective of designing a fuel reduction burning program which minimises the impact on both of these locations.

These results are based on the assumption that the fuel reduction burning is occurring in every source region for every day in the month, which is equivalent to burning occurring on a random selection of days for many realisations of an April month with the same meteorology being present in each realisation. Therefore, Figure 4 represents a risk climatology for smoke impacts in the Oven's Valley region. How this climatology will vary in response to inter-decadal variation of autumn weather conditions is the subject of further investigation.
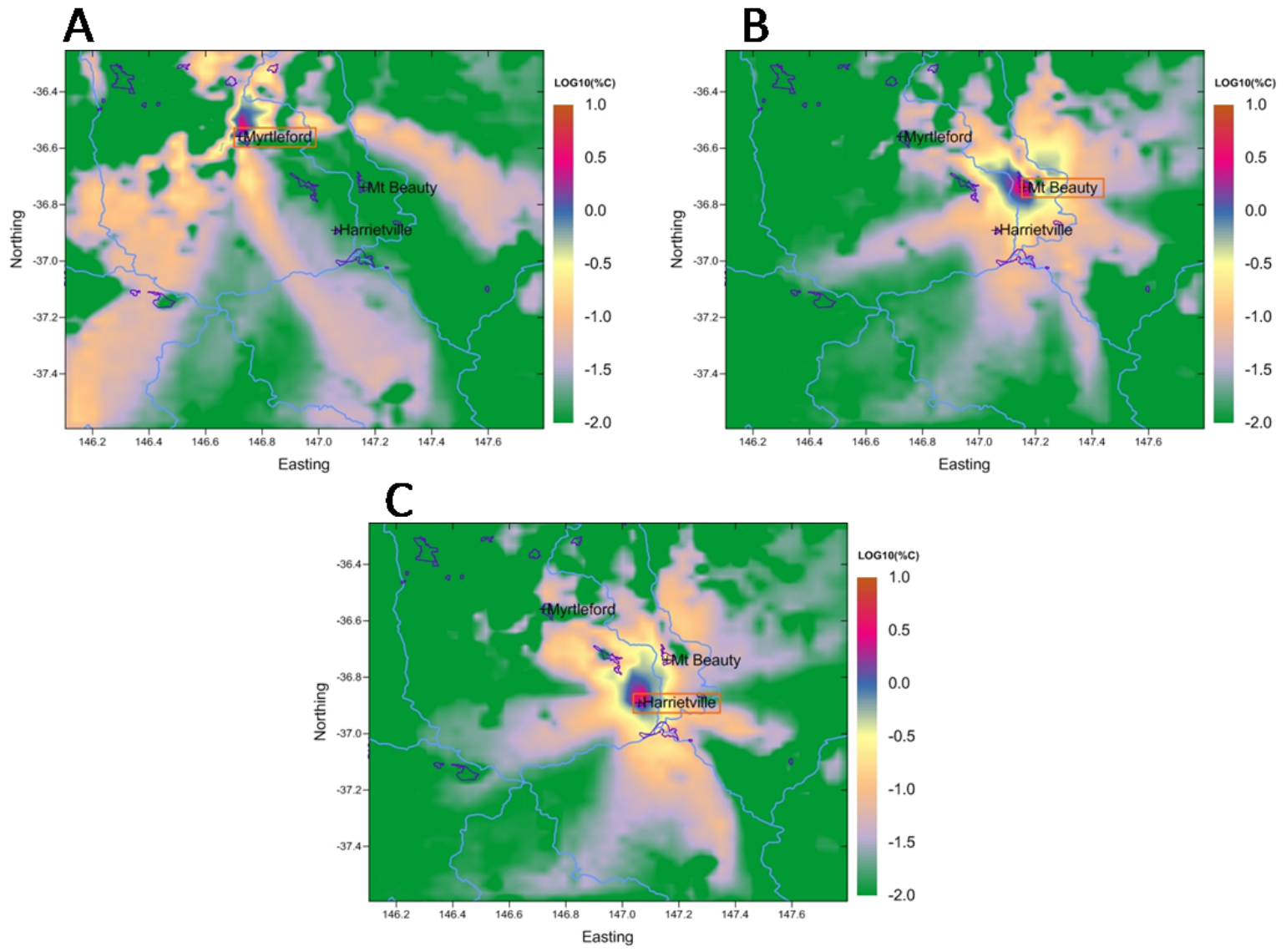

Figure 4. The percentage relative contribution (plotted on a log scale) of smoke emissions released from every $3 \times 3 \mathrm{~km}^{2}$ grid cell in the modelling domain to A: Myrtleford, B: Mt. Beauty, and C: Harrietville.

\section{DISCUSSION AND CONCLUSIONS}

The results presented in the previous section provide an example of how a matrix of modelled sourcereceptor relationships generated by the tagged tracer methodology can be used to generate a risk climatology based on monthly average source impacts. However, there are many other powerful uses of this technique that can be applied to the management of fuel reduction burning. For example, an alternative approach would be to re-sample the model output multiple times, stochastically selecting the days, and the regions that are selected for fuel reduction burning. This can be used to build up a risk-based approach in which fuel reduction burns are only assumed to occur under limited classes of meteorological conditions. This approach 
Meyer et al., Smoke impacts from prescribed burning in Victoria; developing a risk climatology.

could be refined further to include rules-based decisions making- modelled on those currently used by the Agencies to select areas to burn.

An extension of the system to include other months and years is straightforward and will enable the intra- and inter-annual variation of meteorology to be factored into the analysis. Additionally, extension of the system to consider a warming atmosphere can also be undertaken using existing tools.

Finally, the system can also be used to investigate the veracity of various screening approaches (such as using local wind speed and direction) to determine the likelihood of smoke from a fuel reduction burn impacting populated regions. As such, the system described in this paper provides a powerful tool that can be used to investigate and contribute to management systems for undertaking fuel reduction burns which result in minimal risk to nearby rural and urban population centres and sensitive agricultural regions.

\section{REFERENCES}

Cope M. E., Hess, G. D., Lee, S., Tory, K., Azzi, M., Carras, J., Lilley, W., Manins, P. C., Nelson, P., Ng, L., Puri, K., Wong, N., Walsh, S., and Young, M. (2004). The Australian Air Quality Forecasting System. Part I: Project Description and Early Outcomes. J. Appl. Meteorol., 43, 649-662.

DEC (2010). Environment and Conservation News, 6/10, 2010.

Fire Ecology Working Group, (2004). Guidelines and Procedures for Ecological Burning on Public Land in Victoria 2004,. Department of Sustainability and Environment, Melbourne.

Haverd, V., M. R. Raupach1, P. R. Briggs, J. G. Canadell, S. J. Davis, R. M. Law, C. P. Meyer, G. P. Peters C. Pickett-Heaps, and B. Sherman (2013). The Australian terrestrial carbon budget, Biogeosciences, 10, 1-19, doi:10.5194/bg-10-1-2013.

Hurley, P. J., (2009). The Air Pollution Model (TAPM) version 4. Part 1. Technical description. Aspendale, Vic.: CSIRO Atmospheric Research. (CSIRO Atmospheric Research technical paper; 71). 54 p.

Johnston, F.H., Bailie, R.S., Pilotto, L.S., Hanigan, I.C. (2007). Ambient biomass smoke and cardio respiratory hospital admissions in Darwin, Australia, BMC Public Health, 7, 240, doi: 10.1186/14712458-7-24

Kennison, K (2009). Bushfire Generated Smoke Taint in Grapes and Wine, GWRDC Project Number: RD 05/02-3, Department of Agriculture and Food, Western Australia 50 pp.

Meyer, C. P., A. K. Luhar and R. M. Mitchell (2008). Biomass burning emissions over northern Australia constrained by aerosol measurements: I-Modelling the distribution of hourly emissions. Atmospheric Environment, 42(7): 1629-1646.

Meyer, C.P. (Mick), Reisen, F, Keywood, M. and Crumeyrolle, S. (2011). Impacts of smoke from regeneration burning on air quality in the Huon Valley, Tasmania, Final report to Forestry Tasmania, CSIRO Marine and Atmospheric Research, August 2011, 78p.

Meyer, C.P, Cope, M. Lee, S., Young, S. and Keywood, M (2012). Development of accounting and verification methodologies for biomass burning emissions from Australian Savanna Woodlands Final report to Northern Australian Indigenous Land and Sea Alliance ,CSIRO Division of Marine and Atmospheric Research, November 2012, 54p.

Pope, C.R., and Dockery, D.W. (2006). Health Effects of Fine Particulate Air:Pollution: Lines that Connect, J. Air \& Waste Manage. Assoc. 56:709-742.

Teague, B., McLeod, R., and Pascoe, S. (2010). Final Report Summary, 2009 Victorian Bushfires Royal Commission, Parliament of Victoria, 52p.

Wain A., Mills G. (2005). The Australian Smoke Management Forecast System, BMRC Research Report 117, Bureau of Meteorology Melbourne 